\title{
Influence of Coil Pitch on Thermo-Fluid Characteristics for Square Channel Spiral Coil Sub-Cooled Condenser
}

\author{
Hardeep Singh ${ }^{\text {1) }}$ Mitsuhisa Ichiyanagi ${ }^{1)}$ Takashi Suzuki ${ }^{1)}$ \\ 1) Sophia University, 7-1, Kioi-cho Chiyoda-Ku, Tokyo, 102-8554, Japan
}

Received on March24,2019

\begin{abstract}
This research paper describes the effect of coil pitch on thermo-fluids characteristics of the spiral coil sub-cooled condenser. Primarily, the heat transfer results for channel size of $4.1 \mathrm{~mm} \times 4.1 \mathrm{~mm}$ were experimentally found and validated with the CFD analysis. As the satisfactory agreements were obtained with smaller errors, the effect of the different coil pitch with the same height on the heat transfer has been examined by using the CFD analysis. Finally, the coil pitch with $6.2 \mathrm{~mm}$ leads to the higher velocity of secondary flow of $0.05 \mathrm{~m} / \mathrm{s}$, while $13.7 \mathrm{~mm}$ pitch depicts the lower value of $0.03 \mathrm{~m} / \mathrm{s}$ inside the square cross-section of the channel. Corresponding to the specific pitch sizes, the heat transfer coefficient depicts high value for the largest pitch size and least for the smallest pitch.
\end{abstract}

KEY WORDS: Heat fluid, Air conditioner, CFD, Spiral coil sub-cooled condenser, Coil pitch (D1)

\section{Introduction}

Automotive air-conditioner provides good human comfort during transportation. However, this comfort put additional load on the automotive engine and added vehicular emissions. In one of the research, a country like America annually about 7.1 million gallons of gasoline consumed by engines only for the airconditioning system [1]. This massive amount of fuel consumption has made vehicular air conditioning unit as the utmost power consumer of energy after the engine. It is imperative to increase the coefficient of performance of the air-conditioning unit to curb the pollution and vehicular emissions. To achieve this goal, the author paid attention to the sub-cooled condenser. Generally, there are two methods for improving the heat transfer rate: active and passive method. In this paper, the primary focus is on passive technique, i.e., only the geometry change and no need for external power. Patil and Vijaybabu [2] conducted the experiments on twisted tape insertion to understand the effect of the twist ratio on heat transfer augmentation. They concluded that the heat transfer increases with a decrease in twist ratio of twisted tape insertion. Maradiya et al., [3] provide a comprehensive study of passive heat transfer devices including the helically twisted tape type heat exchanger. Garcia et al., [4] investigated and compared the influence of corrugated tubes, dimpled tubes, and wire coil insert on heat transfer enhancement. The author [5] investigated the heat transfer coefficient relationship between the flat/conventional and spiral coil sub-cooled condenser. In our studies, it is found that spiral coil sub-cooled condensers (SCSCC) provide better heat transfer rate comparing to the flat/ conventional type sub-cooled condenser. This is because of the higher heat transfer coefficient on the outer side comparing to the inner side of the wall. Patankar et al., [6] investigated the effects of the Dean number on heat transfer in helically coiled tubes for developing and the fully-developed laminar flow. In the literature review, Lin and Ebadian [7] used the k- $€$ model for investigating heat transfer with curvature ratio and Reynolds number. Kumar et al., [8] investigated the hydrodynamics and heat transfer characteristics of the tube-in-tube helical heat exchanger at the pilot plant scale. Based on the literature review, it is observed that spiral kind of heat exchangers are compact and have a high ability of heat transfer. Moreover, the coil pitch is the function of the centrifugal force that leads to secondary flow. Therefore, it is highly required to further investigate the relationship between coil pitch and secondary flow generation.

The objective of the present study is to investigate the effect of SCSCC coil pitch on the thermal as well as fluid characteristics by using CFD analysis. Previous studies illustrated that very few articles were published on R134a and finned type sub-cooled condenser. Hence, the author investigated thermo-fluid characteristics of SCSCC between the high-temperature R134a refrigerant (inside) and low-temperature air (outside).

\section{Experimental setup and cfd analysis}

\subsection{Experimental setup}

A SCSCC with $4.1 \mathrm{~mm} \times 4.1 \mathrm{~mm}$ cross-section channel with wave fin was used for the experiments. All these experiments were conducted by using various sensors and equipment, which are established inside the laboratory. During the test, the surrounding temperature (outside temperature of SCSCC) was controlled by 
using calorimeter. Figure 1 illustrates the schematic overview of calorimeter as well as the front and side view of the complete assembly of the SCSCC. Four spiral tubes in parallel are connected with the upper and lower header of the SCSCC.
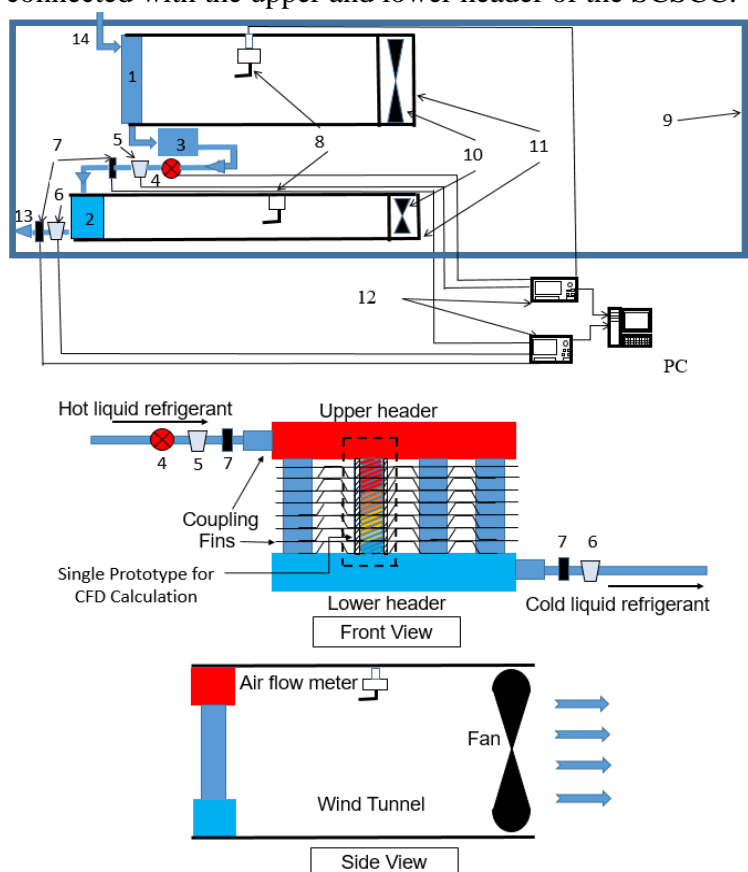

(1) Flat tube condenser (2) Spiral coil sub-cooled condenser (3) Receiver (4) Refrigerant flow meter (5) Inlet pressure gauge (6) Outlet pressure gauge (7) Temperature sensors (8) Velocity measuring nozzles (9) Calorimeter (10) fan (11) Wind tunnels (12) Data Lodgers (13) Refrigerant outlet (14) Refrigerant inlet

Fig. 1 Schematic overview of the calorimeter.

However, only a single spiral tube has been considered for the analysis. Other instruments/ sensors which are used during the bench test has been represented in Fig. 1. Furthermore, the calorimeter is one of the most vital equipments of the bench test and is used to measure the coefficient of performance of the airconditioning unit and is manufactured by Chino Corp. The SCSCC is the primary part of the condenser and works under liquid refrigerant only. In order to convert vapor refrigerant to liquid refrigerant, flat type (conventional type) of the condenser is used. A receiver is installed after flat type condenser, which delivered only liquid refrigerant towards SCSCC (prototype). Moreover, wave type of fins [9] is attached on the outer side of the prototype with the help of brazing. Various measuring sensors and instruments have been described as following in details. The sheathed type thermocouples are used to measure the inlet/ outlet temperatures of the refrigerant as well as air. The T-type thermocouples with model no SUS316 is of Chino Corp with a sensing range of 373 - $423 \mathrm{~K}$. Two wind tunnels separately used for the flat and spiral coil sub-cooled condenser. The fan is manufactured by Oriental motor Corp and have the model number of mdv625/924-24s. Similarly, for measure air velocity, nozzle type velocimeter is used having model name FMA $900 \mathrm{~A}$ and is manufactured by Omega Corp. The refrigerant flow rate is measured by the use of Coriolis type flow meter which has the range from 0 to $20 \mathrm{~L} / \mathrm{min}$. The model number of this mass flow device is FD-SS 20 ASO(3033) and is manufactured by Keyence Corp. The differential type pressure gauge is also installed at the inlet and outlet of the header. The model number of this kind of gauge is PD-23, and it is manufactured by the Sayama Corp. The operating pressure range is $20 \mathrm{kPa}-20 \mathrm{MPa}$. The details of the prototype (SCSCC) has been illustrated in Fig. 2. The crosssectional view illustrates the various fluid galleries (fluid passage), square cross-sectional channel and the fluid volume which is running inside the galleries have been showing in Fig. 2(a), 2(b) and 2(c), respectively.

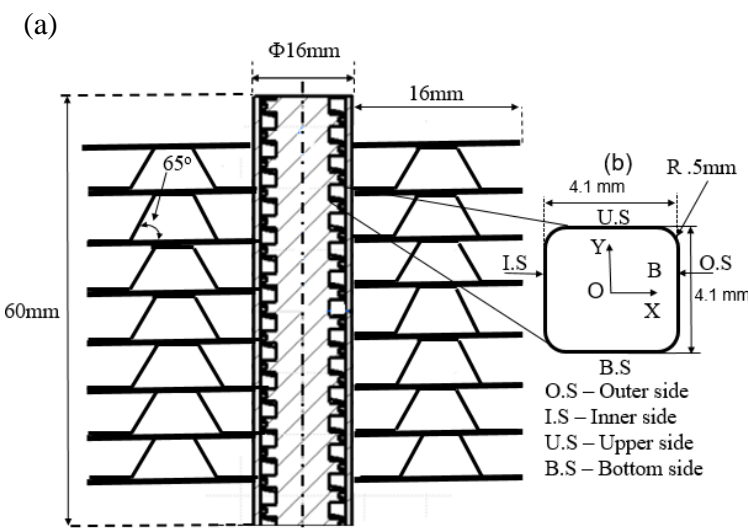

(c)

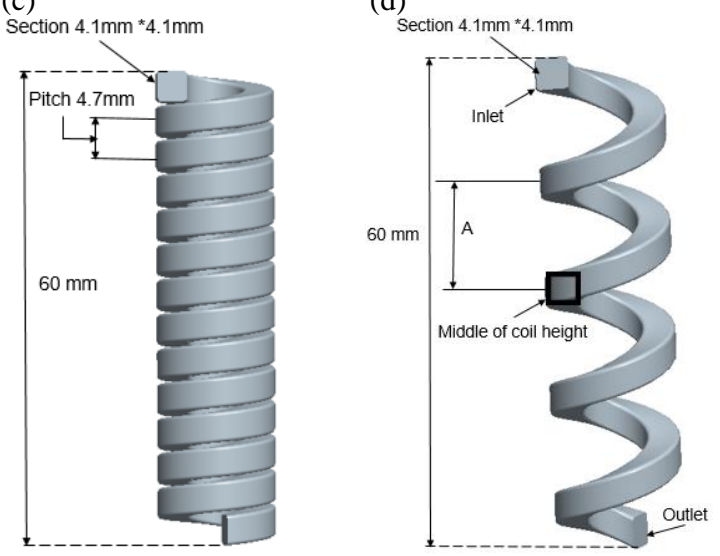

Fig. 2 (a) Cross-section view of spiral coil sub-cooled condenser with $4.1 \mathrm{~mm}$ square channel, (b) channel crosssectional area, (c) inside view of spiral fluid volume which is running through square channels from upper side to lower side and (d) definition of pitch, A, listed in Table2.

Table 1 is representing the experimental as well as CFD boundary conditions for the validation. Similarly, boundary conditions for further CFD study have been represented in Table 2. In Table $1, V_{a}[\mathrm{~m} / \mathrm{s}]$ is the velocity of the air, $\dot{m}_{r}[\mathrm{~kg} / \mathrm{s}]$ is the mass flow rate of the refrigerant, $T_{r_{-} i n}[\mathrm{~K}]$ and $T_{a_{-} i n}[\mathrm{~K}]$ are inlet temperature of refrigerant and air respectively. $P_{r_{-} i n}[\mathrm{MPa}]$ is the 
inlet pressure of the refrigerant. Also, in Table 2, $A[\mathrm{~mm}]$ is the pitch, $a[\mathrm{~mm}]$ is the equivalent pipe diameter, $h[\mathrm{~mm}]$ is the height, and $D[\mathrm{~mm}]$ is the curvature diameter of the prototype. About the straight type heat exchanger, it comprises a straight pipe with a square channel and has flat on the outside. Moreover, in comparison with the spiral heat exchanger, it has the same area ratio resulting in the effect toward airside. As a result, it has the same heat transfer performance as a helical type heat exchanger. $c$ $\left[\mathrm{mm}^{2}\right]$ is the cross-sectional area of the straight type sub-cooled condenser. The 3D axisymmetric model is developed using volume-based computational fluid dynamics (CFD) software ANSYS FLUENT -15. Various grid sizes with cells 6,278,378 (low), 6,683,783 (medium) and 6,756,798 (high) have been used for pitch size $4.7 \mathrm{~mm}$ and examined their effect on simulation results. Grid independence check suggested a final grid size of $6,683,783$ (medium), as it does not show any variation with $6,756,788$ (high) grid size. Moreover, convergence criteria for continuity, momentum, and energy equation is taken as $10^{-3}, 10^{-3}$ and $10^{-3}$ respectively.

Table 1 Experimental conditions of $4.1 \mathrm{~mm} \times 4.1 \mathrm{~mm}$ channel size for refrigerant side

\begin{tabular}{|l|c|c|c|c|c|}
\hline S.No & 1 & 2 & 3 & 4 & 5 \\
\hline$V_{a}[\mathrm{~m} / \mathrm{s}]$ & \multicolumn{5}{|c|}{2.0} \\
\hline$\dot{m}_{r}[\mathrm{~kg} / \mathrm{s}]$ & 0.006 & 0.007 & 0.0075 & 0.008 & 0.0085 \\
\hline$T_{r_{-} \text {in }}[\mathrm{K}]$ & \multicolumn{5}{|c|}{327.6} \\
\hline$T_{a_{-} \text {in }}[\mathrm{K}]$ & \multicolumn{5}{|c|}{1.5} \\
\hline$P_{r_{-} \text {in }}[\mathrm{MPa}]$ & \multicolumn{5}{|c}{} \\
\hline
\end{tabular}

Table 2 CFD condition for variable spiral coil pitch and straight type sub-cooled condenser for refrigerant side

\begin{tabular}{|c|c|c|c|c|c|c|}
\hline $\begin{array}{c}\text { Spiral } \\
\text { S.No }\end{array}$ & 1 & 2 & 3 & 4 & 5 & 6 \\
\hline$A[\mathrm{~mm}]$ & 6.2 & 7.7 & 9.2 & 10.7 & 12.2 & 13.7 \\
\hline$a[\mathrm{~mm}]$ & \multicolumn{6}{|c|}{4.1} \\
\hline$h[\mathrm{~mm}]$ & 60 \\
\hline$D[\mathrm{~mm}]$ & 11.9 \\
\hline $\begin{array}{c}\text { Straight } \\
\text { S. No }\end{array}$ & 1 \\
\hline$c\left[\mathrm{~mm}^{2}\right]$ & $4.1 \times 4.1$ \\
\hline$A[\mathrm{~m}]$ & 0.0 \\
\hline$h[\mathrm{~m}]$ & 60 \\
\hline
\end{tabular}

\subsection{CFD Analysis}

\subsubsection{Governing equations}

The heat transfer rate from the refrigerant is experimentally found and then validated with CFD results at different refrigerant flow rates and constant air velocity. The flow of fluid has been found by using two main governing equations, i.e., Navier-Stokes and continuity equations. Moreover, these are utilized for CFD analysis and has been described below.

$\frac{\partial v}{\partial x}+(v \cdot \nabla) v=-\frac{1}{\rho} \nabla p+\frac{\mu}{\rho} \nabla^{2} \boldsymbol{v}+\boldsymbol{g}$

$\nabla \cdot v=0$

where $\rho\left[\mathrm{kg} / \mathrm{m}^{3}\right]$ is the density, $v[\mathrm{~m} / \mathrm{s}]$ is the flow velocity, $p$ $\left[\mathrm{N} / \mathrm{m}^{2}\right]$ is the pressure, $\mu[\mathrm{Pa} \cdot \mathrm{s}]$ is the dynamic fluid viscosity, and $g\left[\mathrm{~m} / \mathrm{s}^{2}\right]$ is the body accelerations acting on the continuum. For calculating temperatures, the energy equation is utilized and narrates below.

$\frac{\partial(\rho E)}{\partial t}+\nabla \cdot(\overrightarrow{\boldsymbol{v}}(\rho E+p))=\nabla \cdot\left(k_{e f f}\right) \nabla T$

where $E[\mathrm{~J}]$ is the energy of the fluid, $T[\mathrm{~K}]$ is the temperature of a fluid, $k_{\text {eff }}[\mathrm{W} / \mathrm{m} \cdot \mathrm{K}]$ is the effective conductivity.

\subsubsection{Area ratio}

The value of the air-side heat transfer coefficient needs to insert to the boundary condition to obtain the refrigerant thermo-fluid characteristics and other parameters like heat transfer. The actual and CFD geometry is dissimilar because of the complexity of the geometry. Thus, the relationship is established among the real geometry as shown in Fig. 2(a) for $4.1 \mathrm{~mm}$ channel and CFD geometry shown in Fig. 2(c). This relationship is known as area ratio and can be defined as the ratio of the area of the condenser with fins to the condenser area without fins. The heat transfer rate from the spiral coil sub-cooled condenser by CFD, i.e., without fin geometry as shown in Fig. 2 (c), $\dot{Q}_{a 1}[\mathrm{~W}]$ is expressed as below.

$\dot{Q}_{a 1}=A_{a 1} h_{a 1}\left(T_{w}-T_{a}\right)$

Where $A_{a 1}\left[\mathrm{~m}^{2}\right]$ is an area without fins, $h_{a 1}\left[\mathrm{~W} / \mathrm{m}^{2} \mathrm{~K}\right]$ is the heat transfer coefficient without fins, $T_{w}[\mathrm{~K}]$ and $T_{a}[\mathrm{~K}]$ are the wall and air temperatures, respectively. In the same way, the rate of heat transfer from the spiral coil sub-cooled condenser by CFD for a real geometry with fins as shown in Fig. 2 (a), $\dot{Q}_{a 2}[\mathrm{~W}]$, is termed as following

$\dot{Q}_{a 2}=A_{a 2} h_{a 2}\left(T_{w}-T_{a}\right)$

Where $A_{a 2}\left[\mathrm{~m}^{2}\right]$ is the area with fins, $h_{a 2}\left[\mathrm{~W} / \mathrm{m}^{2} \mathrm{~K}\right]$ is the heat transfer coefficient with a fin. Since it is equal to, the heat transfer coefficient of airside represents as following.

$h_{a 1}=\frac{A_{a 2}}{A_{a 1}} h_{a 2}$

From equation (6), the area ratio is determined by using CAD software and heat transfer coefficient $\left(h a_{2}\right)$ firstly determined, and these values are used to obtain $h a_{1}$. 
The empirical relationship of heat transfer for the refrigerant side $\dot{Q}_{r}^{\prime}$ can be written as:

$\dot{Q}_{r}^{\prime}=\dot{m}_{r} C_{p}\left(T_{r_{-} \text {in }}-T_{r_{-} \text {out }}\right)$

where $C_{p}[\mathrm{~kJ} / \mathrm{kg} \cdot \mathrm{K}]$ is the specific heat of refrigerant under the steady-state condition and $T_{r_{-} \text {out }}[\mathrm{K}]$ is the refrigerant outlet temperature. Air and refrigerant sides have different operating conditions, so that different viscous model is chosen for both of them. Tables 3 and 4 illustrate the viscous model as a well iterative solution method for the airside and refrigerant side, respectively.

Table 3 Airside viscous model and approach for the iterative solution method

\begin{tabular}{|c|c|}
\hline Viscous Model & Laminar \\
\hline Solution Method & Simple \\
\hline Gradient & Least Squares Cell-Based \\
\hline Pressure & Second Order \\
\hline Momentum & Second Order Upwind \\
\hline Turbulent Kinetic Energy & First Order Upwind \\
\hline
\end{tabular}

Table 4 Refrigerant side viscous model and approach for the iterative solution method

\begin{tabular}{|c|c|}
\hline Viscous Model & RNG k- $\varepsilon$ \\
\hline Near-wall Treatment & Non-equilibrium \\
\hline Solution Method & Simple \\
\hline Gradient & Least Squares Cell-Based \\
\hline Pressure & Second Order \\
\hline Momentum & Second Order Upwind \\
\hline Turbulent Kinetic Energy & First Order Upwind \\
\hline
\end{tabular}

\section{Results and discussions}

3.1 Experimental results and evaluation of measurement uncertainty

Under the experimental conditions shown in Table 1, the various temperatures and pressures were measured, and their results were summarized in Table 5. The experimental data shown in Table 5 were used as the boundary condition for the CFD calculation described in Section 2.2

The measurement uncertainties at $95 \%$ confidence intervals, URSS, of the temperature measurement of air, temperature measurement of refrigerant and pressure measurement were evaluated using the following equations.

$$
\begin{aligned}
& B_{m}=\left(B_{1}^{2}+B_{2}^{2}\right)^{1 / 2} \\
& U_{R S S}=\left(B_{m}^{2}+\left(t_{s} \sigma_{m}\right)^{2}\right)^{1 / 2}
\end{aligned}
$$

where $B_{n}(\mathrm{n}=1,2,3,4)$ is the bias limit. The value of ts based upon the student's t-test which is 1.96 under the present experimental conditions. The uncertainty due to total measurement errors, $U_{R S S}$, is given by the summation of the bias index, $B_{m}$, and the precision index, $S_{m}$. $U_{R S S}$ of temperature measurement of air and refrigerant, and pressure measurement are $0.79,0.65$ and 0.23 , respectively. Tables 6 (a), (b) and (c) illustrate the relationship among error factors and accuracy for temperature measurement of air, refrigerant and pressure, respectively.

Table 5 Experimental results and boundary condition of SCSCC for refrigerant side CFD analysis with a variable refrigerant flow rate

\begin{tabular}{|l|c|c|c|c|c|}
\hline \multicolumn{1}{|c|}{ S.No } & $\mathbf{1}$ & $\mathbf{2}$ & $\mathbf{3}$ & $\mathbf{4}$ & $\mathbf{5}$ \\
\hline$V_{a}[\mathrm{~m} / \mathrm{s}]$ & 2.0 & 2.0 & 2.0 & 2.0 & 2.0 \\
\hline$T_{a_{-} \text {in }}[\mathrm{K}]$ & 313.0 & 313.0 & 313.0 & 313.0 & 313.0 \\
\hline$T_{a_{-} \text {out }}[\mathrm{K}]$ & 315.7 & 315.9 & 316.0 & 316.1 & 316.5 \\
\hline$\dot{m}_{r}[\mathrm{~kg} / \mathrm{s}]$ & 0.006 & 0.007 & 0.0075 & 0.008 & 0.0085 \\
\hline$T_{r_{-} \text {in }}[\mathrm{K}]$ & 326.1 & 327.6 & 328.1 & 328.6 & 329.8 \\
\hline$T_{r_{-} \text {out }}[\mathrm{K}]$ & 325.1 & 326.6 & 327.1 & 327.6 & 328.7 \\
\hline $\begin{array}{c}P_{r_{-} \text {in }} \\
{[\mathrm{Mpa}]}\end{array}$ & 1.4263 & 1.4783 & 1.4982 & 1.5135 & 1.5587 \\
\hline $\begin{array}{c}P_{r_{-} \text {out }} \\
{[\mathrm{Mpa}]}\end{array}$ & 1.4143 & 1.4643 & 1.4823 & 1.4992 & 1.5332 \\
\hline
\end{tabular}

Table 6 Measurement uncertainties of (a) temperature measurement of air, (b) temperature measurement of refrigerant and (c) pressure measurement

(a)

\begin{tabular}{|c|c|c|c|c|}
\hline S.No & Error factor & Units & Symbol & Accuracy \\
\hline 1 & $\begin{array}{c}\text { Thermo-couple } \\
\text { error }\end{array}$ & {$[\mathrm{K}]$} & $B_{1}$ & $\pm 0.75 \%$ \\
\hline 2 & $\begin{array}{c}\text { Airflow tester } \\
\text { error }\end{array}$ & {$[\mathrm{K}]$} & $B_{2}$ & $2.215(2 \%)$ \\
\hline 3 & Bias index & {$[\mathrm{K}]$} & $B_{m}$ & 0.33 \\
\hline 4 & $\begin{array}{c}\text { Standard } \\
\text { deviation }\end{array}$ & {$[\mathrm{K}]$} & $\sigma_{1}$ & 0.367 \\
\hline 5 & Precision index & {$[\mathrm{K}]$} & $\sigma_{m}$ & 0.367 \\
\hline
\end{tabular}

(b)

\begin{tabular}{|c|c|c|c|c|}
\hline S.No & Error factor & Units & Symbol & Accuracy \\
\hline 1 & $\begin{array}{c}\text { Thermo-couple } \\
\text { error }\end{array}$ & {$[\mathrm{K}]$} & $B_{1}$ & $\pm 0.75 \%$ \\
\hline 2 & $\begin{array}{c}\text { Refrigerant } \\
\text { flow meter }\end{array}$ & {$[\mathrm{K}]$} & $B_{3}$ & $\begin{array}{c}0.00736 \\
(1 \%)\end{array}$ \\
\hline 3 & Bias index & {$[\mathrm{K}]$} & $B_{m}$ & 0.33 \\
\hline 4 & $\begin{array}{c}\text { Standard } \\
\text { deviation }\end{array}$ & {$[\mathrm{K}]$} & $\sigma_{2}$ & 0.1496 \\
\hline 5 & Precision index & {$[\mathrm{K}]$} & $\sigma_{m}$ & 0.1496 \\
\hline
\end{tabular}

(c)

\begin{tabular}{|c|c|c|c|c|}
\hline S.No & Error factor & Units & Symbol & Accuracy \\
\hline 1 & $\begin{array}{c}\text { Pressure gauge } \\
\text { error }\end{array}$ & {$[\mathrm{MPa}]$} & $B_{4}$ & $\pm 0.5 \%$ \\
\hline 2 & Bias index & {$[\mathrm{MPa}]$} & $B_{m}$ & \pm 0.0078 \\
\hline 3 & $\begin{array}{c}\text { Standard } \\
\text { deviation }\end{array}$ & {$[\mathrm{MPa}]$} & $\sigma_{3}$ & 0.118 \\
\hline 4 & Precision index & {$[\mathrm{MPa}]$} & $\sigma_{m}$ & 0.118 \\
\hline
\end{tabular}


The CFD and experimental results for heat transfer rate and refrigerant temperature difference have been discussed in Tables 7 and 8 , respectively. The experimental results for the prototype has been verified with CFD results. Following tables depicts the maximum and average error of $8.5 \%$ and $2.0 \%$ respectively among the experimental and CFD results. Hence, further CFD results can be proceeded for further CFD investigation.

Table 7 Comparison of CFD and experimental heat transfer rate with different mass flow rate toward the refrigerant side

\begin{tabular}{|c|c|c|c|c|}
\hline S.No & $\begin{array}{c}\text { Experimental } \\
\text { heat transfer rate, } \\
q[\mathrm{~W}]\end{array}$ & $\begin{array}{c}\text { CFD heat transfer } \\
\text { rate, } q[\mathrm{~W}]\end{array}$ & \multicolumn{2}{|c|}{$\begin{array}{c}\text { Error ratio } \\
{[\%]}\end{array}$} \\
\hline 1 & 14.0 & 15.2 & Max & 8.5 \\
\hline 2 & 12.4 & 12.6 & Avg & 2.0 \\
\hline
\end{tabular}

Table 8 Comparison of CFD and experimental temperature difference with different mass flow rate toward the refrigerant side

\begin{tabular}{|c|c|c|c|c|}
\hline S.No & $\begin{array}{c}\text { Experimental } \\
\text { refrigerant } \\
\text { temperature } \\
\text { difference, } \\
T_{r_{\text {_in }}-T_{r_{-} \text {out }}[\mathrm{K}]}\end{array}$ & $\begin{array}{l}\text { CFD refrigerant } \\
\text { temperature } \\
\text { difference, } \\
T_{r_{-} \text {in }}-T_{r_{-} \text {out }}[\mathrm{K}]\end{array}$ & \multicolumn{2}{|c|}{$\begin{array}{c}\text { Error ratio } \\
{[\%]}\end{array}$} \\
\hline 1 & 1.1 & 1.2 & Max & 9.0 \\
\hline 2 & 1.0 & 1.1 & Avg & 10.0 \\
\hline
\end{tabular}

\subsection{Analysis of variable spiral coil pitch by CFD investigation}

Figure 4 exemplifies the evidence regarding the generation of induced secondary flow inside the channel and its dependability on the spiral coil pitch. The pitch is varied from $6.2 \mathrm{~mm}$ to $13.7 \mathrm{~mm}$, the cross-sectional channel along contour figures of secondary flow generation has been represented in Figs. 4 (a) to 4 (f). Besides,this figure revealed the effect of spiral coil pitch on the secondary flow generation. It is clearly showing from Fig. 4(a) that two vortices with high velocities formed at the bottom and upper sides of the channel. In contrast in Fig. 4(f) the size of the vortices get declined, and high-velocity refrigerant shifted from the outer side towards the core of the channel as the spiral coil pitch is increasing. Conceivably centrifugal force is the reason behind the weak generation of secondary flow. As the spiral coil pitch increased, the primary flow velocity increases and reduces the effect of centrifugal force.

The relationship among secondary velocity magnitude (induced velocity) along distance y has been illustrated in fig. 5 . The analysis has been made at mid of the coil for assumed as a developing flow. Results provide good relationship among secondary velocity and channel distance except for the pitch size $7.7 \mathrm{~mm}$. The spiral coil with the lowest pitch of $6.2 \mathrm{~mm}$ provides the highest generation of secondary velocity with the magnitude of $0.05 \mathrm{~m} / \mathrm{s}$ in the cross-sectional channel. Centrifugal force considered as the reason behind this effect, which induced because of the smaller spiral coil pitch. Correspond to the largest pitch, i.e., in $13.7 \mathrm{~mm}$ there is a weak generation of secondary velocity with the magnitude scale of $0.03 \mathrm{~m} / \mathrm{s}$.

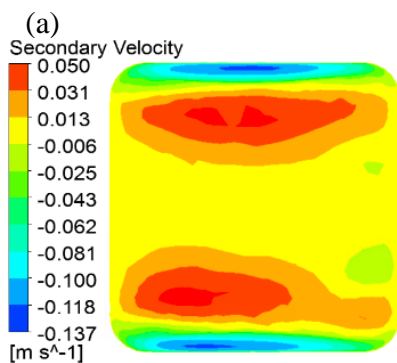

(c)
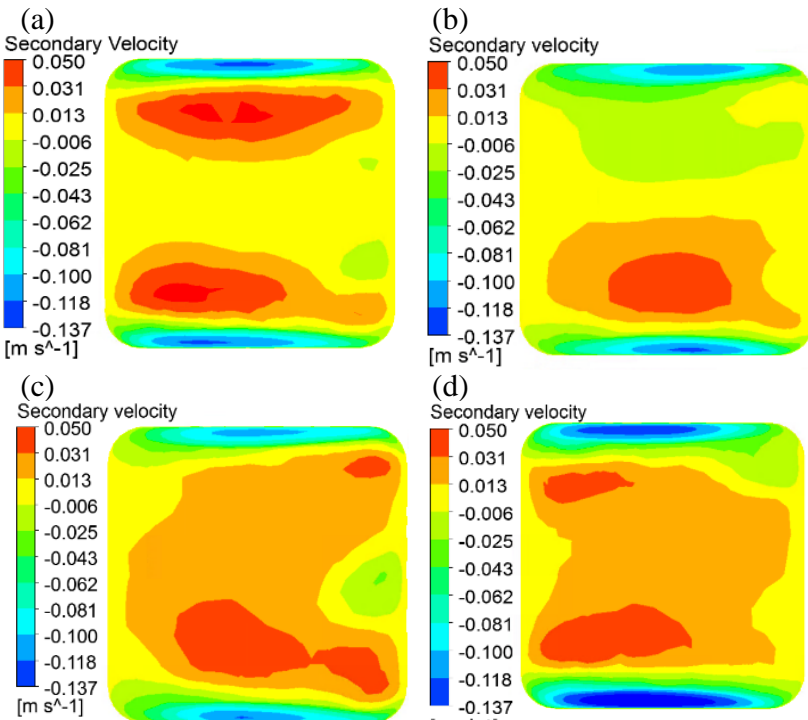

(d)

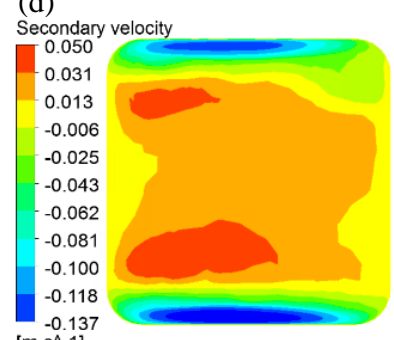

(e)

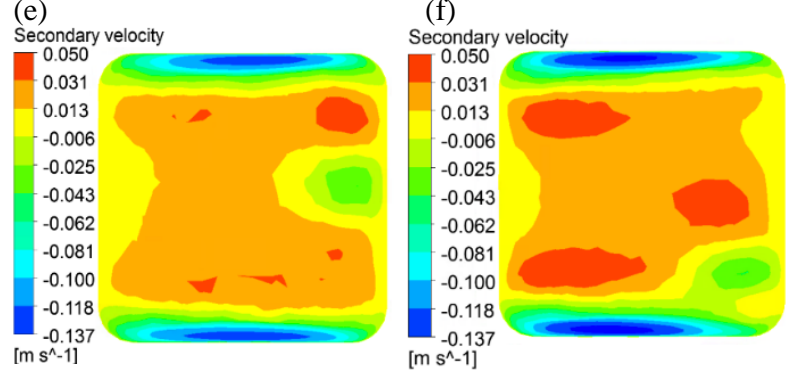

Fig. 4 Refrigerant velocity distribution along Y direction inside the square cross-section channel of SCSCC at the middle height of the coil. Spiral coil pitch is (a) $6.2 \mathrm{~mm}$, (b) $7.7 \mathrm{~mm}$, (c) 9.2 $\mathrm{mm}$, (d) $10.7 \mathrm{~mm}$, (e) $12.2 \mathrm{~mm}$ and (f) $13.7 \mathrm{~mm}$.

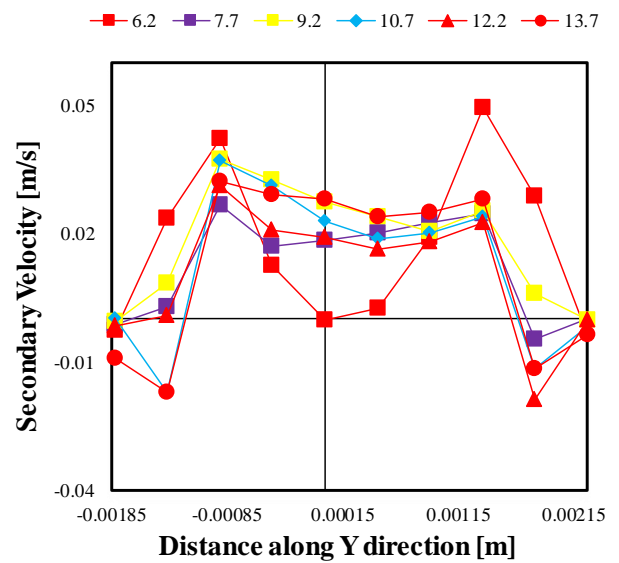

Fig. 5 Refrigerant velocity magnitude distribution along Y direction inside the square cross-section channel of SCSCC at the middle height of the coil. Spiral coil pitch is (a) $6.2 \mathrm{~mm}$, (b) $7.7 \mathrm{~mm}$, (c) $9.2 \mathrm{~mm}$, (d) $10.7 \mathrm{~mm}$, (e) $12.2 \mathrm{~mm}$ and (f) 13.7 $\mathrm{mm}$. 


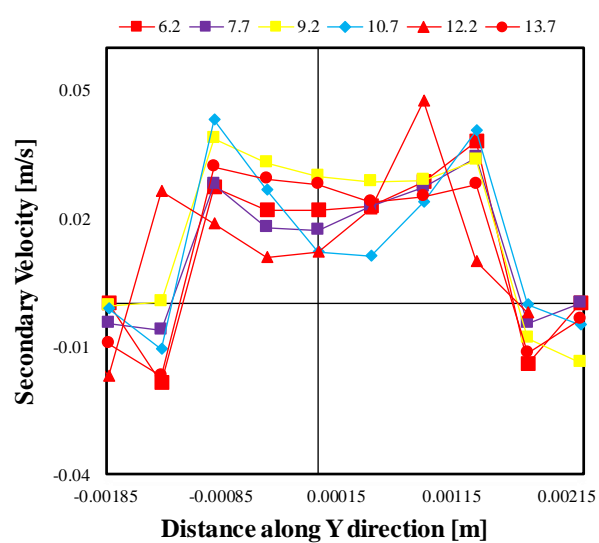

Fig. 6 Refrigerant velocity magnitude distribution along Y direction inside the square cross-section channel of SCSCC at the outlet of the coil. Spiral coil pitch is (a) $6.2 \mathrm{~mm}$, (b) $7.7 \mathrm{~mm}$ (c) $9.2 \mathrm{~mm}$, (d) $10.7 \mathrm{~mm}$, (e) $12.2 \mathrm{~mm}$ and (f) $13.7 \mathrm{~mm}$.

The extreme positions for secondary velocity magnitude at the outer and inner side of the channel are showing at positions of $0.00115 \mathrm{~m}$ and $-0.00085 \mathrm{~m}$ respectively. In this case, the centrifugal force decreased with an increase in the pitch size.

(a)

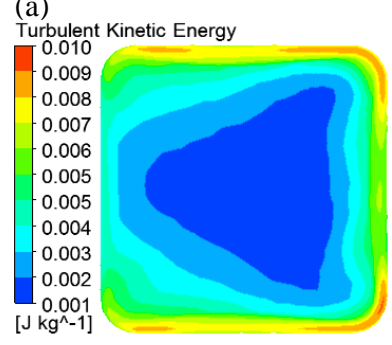

(c)

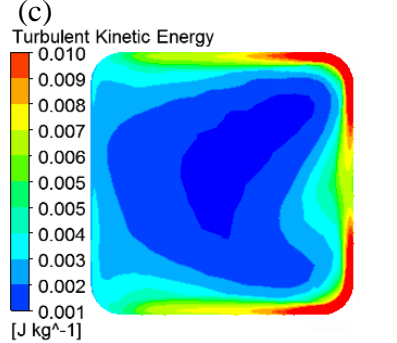

$\left[\mathrm{J} \mathrm{kg}{ }^{\wedge}-1\right]$

(e)

Turbulent Kinetic Energy

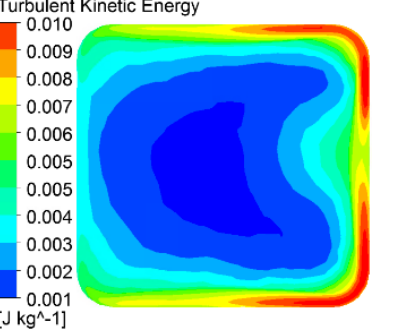

[J $\left.\mathrm{kg}^{\wedge}-1\right]$

Fig. 7 Refrigerant turbulent kinetic energy distribution along $\mathrm{X}$ direction inside square cross-section channel of SCSCC at middle height of coil. Spiral coil pitch is (a) $6.2 \mathrm{~mm}$, (b) 7.7 $\mathrm{mm}$, (c) $9.2 \mathrm{~mm}$, (d) $10.7 \mathrm{~mm}$, (e) $12.2 \mathrm{~mm}$ and (f) $13.7 \mathrm{~mm}$.

Fully develop flow is the function of coil length; therefore, the analysis was examined at the outlet of the coil for various coil pitches and has been illustrated in figure 6 . The second and third smallest spiral coil pitch (12.2 mm and $10.7 \mathrm{~mm})$ shows the higher generation of secondary velocity with a magnitude of about 0.05 $\mathrm{m} / \mathrm{s}$ and $0.045 \mathrm{~m} / \mathrm{s}$, respectively. In these spiral coil pitch cases, the centrifugal force may be depleted, but the resistance to flow (friction force) possibly declined, and results of both the forces lead to the high generation of secondary velocity. While, in case of $13.7 \mathrm{~mm}$ pitch coil, the mainstream flow is very high and subsequently generated weak centrifugal force. Moreover, in case of lower pitch $(6.2 \mathrm{~mm})$ feasibly there is a higher generation of centrifugal force, but higher fluid contact length offered the eminent wall fluid friction. In such circumstances, the centrifugal force credible overcame by the frictional force and generated the least secondary flow effect.

Turbulent kinetic energy, which is one of the significant components for heat transfer coefficient has been illustrated in Fig. 7. From Figs. 7(a) to 7(f), represent the behavior of turbulent kinetic energy on the pitch of the coil. Figure 7(a) depicts a weak fluctuation of turbulent kinetic energy towards the outer side of the channel. Higher contact surface (friction) must be the reason behind the generation of lower turbulent kinetic energy.

However, on the other hand in Fig. 7(f), i.e., a pitch is increasing, there is a high fluctuation of velocity, i.e. (turbulent kinetic energy) is getting higher towards the outer side of the coil as shown in Fig. 2(b) and hence leads to a high heat transfer coefficient. Prominent, because of the lower surface friction that is reduced by increasing the coil pitch.

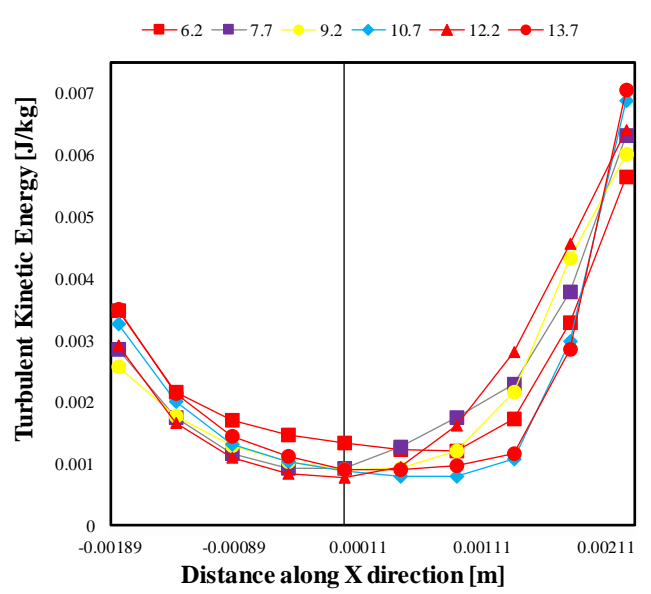

Fig. 8 Refrigerant turbulent kinetic energy magnitude distribution along $\mathrm{X}$ direction inside the square cross-section channel of SCSCC at the middle height of the spiral coil. Spiral coil pitch is (a) $6.2 \mathrm{~mm}$, (b) $7.7 \mathrm{~mm}$, (c) $9.2 \mathrm{~mm}$, (d) $10.7 \mathrm{~mm}$, (e) $12.2 \mathrm{~mm}$ and (f) $13.7 \mathrm{~mm}$.

The magnitude of turbulent kinetic energy along crosssectional channel distance for various spiral coil pitches has been 
represented in Fig. 8. The results were examined at the middle height of the spiral coil. The figure revealed that large spiral coil pitch leads to the higher generation of turbulent kinetic energy on the outer side $(0.00211 \mathrm{~m})$ as compared to the inner side (-0.00189 $\mathrm{m})$ of the spiral coil channel as shown in Fig. 8. Surface friction could be the reason behind this effect. As the contact surface area increased, the fluctuation of velocity is decreasing that leads to lower turbulent kinetic energy.

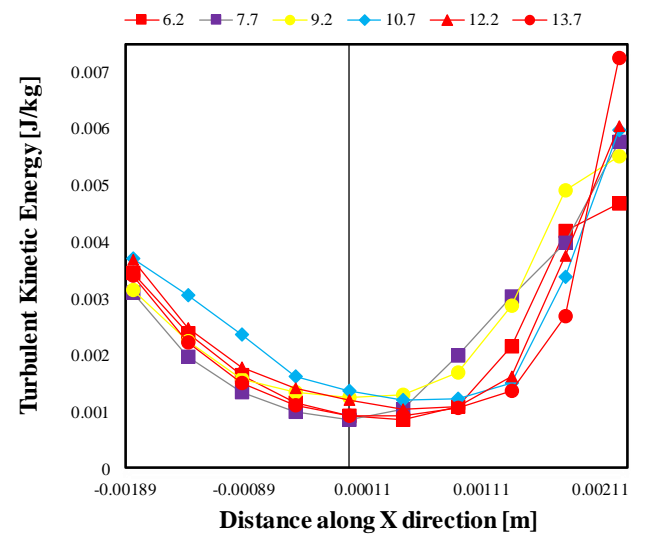

Fig. 9 Refrigerant turbulent kinetic energy magnitude distribution along $\mathrm{X}$ direction inside the square cross-section channel of SCSCC at the outlet of the spiral coil. Spiral coil pitch is (a) $6.2 \mathrm{~mm}$, (b) $7.7 \mathrm{~mm}$, (c) $9.2 \mathrm{~mm}$, (d) $10.7 \mathrm{~mm}$, (e) $12.2 \mathrm{~mm}$ and (f) $13.7 \mathrm{~mm}$.

Similarly, like the secondary flow generation, the turbulent kinetic energy also observed at the outlet section and results has been represented in Fig. 9. The results again show the high magnitude of turbulent kinetic energy for the largest and least for the smallest spiral coil pitch. Also, in this case, the turbulent kinetic energy is higher towards the distance of $0.00211 \mathrm{~m}$ as compared to the $-0.00189 \mathrm{~m}$ of the channel.

\subsection{Heat transfer coefficient relationship with Dean number}

The effect of a spiral coil pitch on the refrigerant heat transfer coefficient has been examined and illustrates in Fig. 10. Dean number, De [-], is a term of spiral coil pitch has been calculated by the following equation 10. It has been shown in the figure that the heat transfer coefficient is increased concerning increasing the pitch. The reason behind this result is the turbulent kinetic energy. The smallest spiral coil pitch expects to dispense high friction because of the high contact surface area in contrast of larger spiral coil pitch, it is estimated as lower friction, and that leads to the higher fluctuation of refrigerant velocity (turbulent kinetic energy) towards the outer side of the coil.

$$
D e=\operatorname{Re} \sqrt{\delta}
$$

$$
\delta=\frac{2 a D}{D^{2}+4 A^{2}}
$$

$R e=\frac{\rho v d}{\mu}$ is the Reynolds number

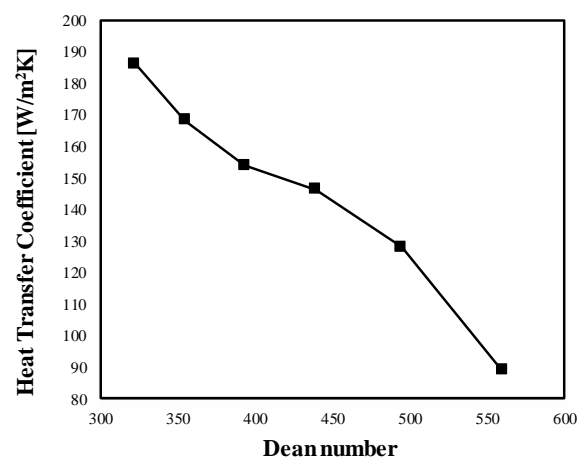

Fig. 10 Effect of Dean number on the average value of heat transfer coefficient (a)

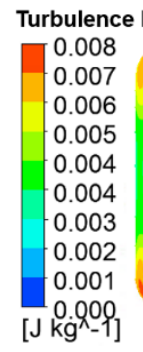

(b) Turbulence Kinetic Energy 0.008 0.008
0.006
0.005
0.004
0.004
0.003
0.002
0.001
0.000
$\left[\mathrm{~J} \mathrm{~kg}_{-}{ }^{2}\right]$
Fig. 11 Refrigerant turbulent kinetic energy distribution contour along $\mathrm{X}$ direction inside square cross-section channel (a) SCSCC and (b) straight type sub-cooled condenser.

Figure 11 shows the contour figure of turbulent kinetic energy among the largest pitch spiral coil and straight tube sub-cooled condenser. The analysis was carried out at the same Reynolds number as well as the same dimensions as the spiral coil have been taken into account as shown in Table 2. From the figures, it is clearly shown that the turbulent kinetic energy is higher towards the outer side relating to the inner side in the case of the spiral coil. Moreover, the fluctuation of refrigerant velocity (turbulent kinetic energy) in the spiral coil is higher as compared to the straight type sub-cooled condenser. This is because of the secondary flow that generates inside the spiral coil.

The magnitude of turbulent kinetic energy among the highest pitch spiral and straight type sub-cooled has been shown in Fig. 12. At the central cross-section channel $(0.0011 \mathrm{~m})$, the turbulent kinetic energy shows high intensity for straight type sub-cooled condenser as comparing to the SCSCC. However, toward the outer side of the coil $(0.00211 \mathrm{~m})$, turbulent kinetic energy depicts higher values for SCSCC among straight type sub-cooled condenser. 
Table 9 illustrates the relationship among spiral coil pitch/straight type sub-cooled condenser and refrigerant side heat transfer coefficient. It is clear from the table that straight type subcooled condenser illustrates the lower value of heat transfer coefficient compared to the SCSCC with varied pitch sizes.

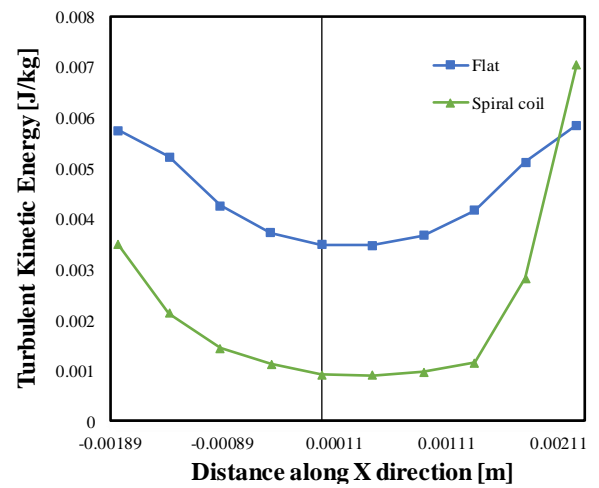

Fig. 12 Refrigerant turbulent kinetic energy magnitude distribution across the channel for SCSCC and straight type sub-cooled condenser.

Table 9 Refrigerant side heat transfer coefficient for varied spiral coil pitch and straight sub-cooled condenser

\begin{tabular}{|c|c|c|}
\hline S.No & Pitch A & $\mathrm{HTC}\left(\mathrm{W} / \mathrm{m}^{2} \cdot \mathrm{K}\right)$ \\
\hline 1 & 6.2 & 88.7 \\
\hline 2 & 7.7 & 127.9 \\
\hline 3 & 9.2 & 146.1 \\
\hline 4 & 10.7 & 153.8 \\
\hline 5 & 12.2 & 168.3 \\
\hline 6 & 13.7 & 186.1 \\
\hline 7 & Straight & 40.0 \\
\hline
\end{tabular}

\section{CONCLUSIONS}

This paper reveals the effect of coil pitch on thermo-fluid characteristics by using a CFD tool. Firstly, the rate of heat transfer numerically investigated and these results were validated by comparison with experimental data. Good agreements were found during the comparison, and further CFD analysis was carried out. As a result, the following things have been clear.

- The investigation revealed the effect of spiral coil pitch on secondary flow generation.

- Larger the pitch least the induced secondary velocity in the spiral coil. Smaller pitch leads to higher secondary velocity as it intensifies the centrifugal force.

- Turbulent kinetic energy towards the outer side of the SCSCC is increasing with an increase in the pitch. Besides, in the least pitch case, the turbulent kinetic energy is greater than the higher coil pitch because it overcomes the effect of friction as secondary velocity is maximum compared to the other pitch sizes.
- In comparison among high coil pitch and the straight type sub-cooled condenser, there is high turbulent kinetic energy as compared to the straight type subcooled condenser. Moreover, this high turbulent kinetic energy shows high magnitude towards the outer side of the coil.

This paper is written based on a proceeding presented at the 2019 JSAE Congress held at Yokohama from 22 May to 24 May 2019.

\section{REFERENCES}

[1] Johnson VH.: Fuel used for vehicle air conditioning: a state-by-state thermal comfort-based approach (National Renewable Energy Laboratory, Society of Automotive Engineers Inc.), SAE paper, no 2002-011957, 2002, doi:10.4271/2002-01-1957.

[2] Patil, S.V., Vijaya Babu, P.V., M., Heat transfer enhancement through a square duct fitted with twisted tape inserts. Heat and Mass Transfer/ WaermeundStoffuebertragung 48(10), 1803-1811, 2012.

[3] Maradiya, C., Vadher, J., Agarwal, R., The heat transfer enhancement techniques and their thermal performance factor, Beni-Suef University Journal of Basic and Applied Sciences, 7(1), pp.1-21, (2018).

[4] Garcia, A et al., The influence of artificial roughness shape on heat transfer enhancement: corrugated tubes, dimple tubes and wire coils. Appll. Therm. Eng. 35(1), 196-201, 2012.

[5] Singh, H., Suzuki, T., Washiashi, J., Ichiyanagi, M. et al.,(2018) Influence of Secondary Flow Generation on Heat Transfer inside the Fin Type Spiral Sub-Cooled Condenser by Experimental and CFD Analysis, SAE Technical Paper 2018-32-0054, doi.org/10.4271/201832-0054.

[6] Hawe, W.B., Some Sidelights on the heat transfer problems, Transactions of the institution of chemical engineering, 32, pp. 161-167, 1932.

[7] S.V. Patankar., V.S. Pratap., D.B. Spalding., (1974) Prediction of laminar flow and heat transfer in helically coiled pipes, J. Fluid Mech. 62 53e551.

[8] C.X. Lin., M.A. Ebadian., (1997) Developing turbulent convective heat transfer in helical pipes, Int. J. Heat Mass Transf. 40 3861e3873.

[9] Samana, T., Kiatsiriroat, T., Nuntaphan, A., (2014) Enhancement of fin efficiency of a solid wire fin by oscillating heat pipe under forced convection, Case Studies in Thermal Engineering, 2, pp36-41. 